\title{
虫草素的全合成研究
}

\author{
李启欢阳如春阮志忠胡蹈而海新肖 强* \\ (江西科技师范大学 江西省有机功能分子重点实验室 南昌 330013)
}

\begin{abstract}
摘要 虫草素具有广谱的生物活性和药理学作用, 有着重要的开发价值. 为了大量制备高纯度虫草素, 分别以 $D$-葡萄 糖和 $D$-木糖为原料, 以 Barton-McCombie 反应脱去葡糖糖和木糖 $3^{\prime}$-手基合成得到 $3^{\prime}$-脱氧核糖为关键步骤, 分别经过 8 步和 7 步化学反应以 $37 \%$ 和 $40 \%$ 的总产率完成了虫草素的全合成, 产物纯度 $>98.5 \%$.
\end{abstract}

关键词 核苷; 全合成; 天然产物; 糖基化; 自由基反应; 碳水化合物

\section{Total Synthesis of Cordycepin}
Li, Qihuan Yang, Ruchun Ruan, Zhizhong
$\mathrm{Hu}, \mathrm{TaO}$
Ding, Haixin
Xiao, Qiang*
(Jiangxi Key Laboratory of Organic Chemistry, Jiangxi Science \& Technology Normal University, Nanchang 330013)

\begin{abstract}
Cordycepin has a large spectrum of biological and pharmaceutical activities, which is beneficial to human health in a number of aspects. In present paper, two total synthetic routes are developed to provide high quality product of cordycepin using $D$-glucose or $D$-xylose as the starting materials. The key step of the total synthesis is deoxygenation of 3-OH using Barton-McCombie reaction to afford 3-deoxyribose. Cordycepin is obtained in 8 steps and 7 steps with $37 \%$ and $40 \%$ overall yield, respectively. Its purity is above $98.5 \%$.
\end{abstract}

Keywords nucleoside; total synthesis; natural product; glycosylation; radical reactions; carbohydrate

虫草素(3'-脱氧腺苷, Cordycepin, 1, 图 1)是从蛹虫 草(Cordyceps sinensis) 中分离得到的天然核苷化合物 ${ }^{[1]}$, 研究表明该化合物具有广谱的生物活性和药理学作用, 包括抗癌、抗菌、消炎、抗病毒和抗肺细胞纤维化等 ${ }^{[2]}$. 特别是近年来生物学研究进一步发现, 虫草素可能具有 增强人类免疫力 ${ }^{[3]}$ 和清除因人老龄化引起的体内自由基 增加的功效 ${ }^{[4]}$, 引起了科学家的广泛研究兴趣.

目前虫草素的获取, 主要是通过从人工培育的蛹虫 草中提取分离获得, 制备成本高, 价格昂贵. 化学合成 主要是以腺苷为原料(图 1), 采用两种方法半合成得到 虫草素, 第一种方法是通过多步骤的基团保护操作, 单 独裸露出 3'位的羟基, 接着用 Barton-McCombie 自由基 还原反应去除 $3^{\prime}$ 位的羟基, 然后脱去保护基团得到目标 产物 ${ }^{[5]}$; 第二种方法是通过乙酰溴和腺苷反应得到 $2^{\prime}$ (3') 澳的腺苷乙酸酯混合物, 然后加氢还原去除溴原子, 脱去保护基团后进一步分离得到目标产物 ${ }^{[6]}$.

为了获取公斤级的虫草素用于生物活性研究, 在重
复第二种方法时我们发现，在加氢反应去除溴原子时会 有 $2 \% \sim 3 \%$ 左右的碱基消除反应从而产生副产物腺嘌 呤, 该副产物很难从虫草素产品中除去, 严重影响了虫 草素的纯度. 进而我们考虑通过全合成的方法进行虫草 素的制备, 文献中其完整的全合成方法报道很少 ${ }^{[7]}$. 本 文分别以廉价易得的 $D$-葡萄糖和 $D$-木糖为原料, 经过 分别经过 8 步反应和 7 步反应完成了虫草素的全合成, 产品纯度大于 $98.5 \%$.

\section{1 结果与讨论}

从反合成分析(图 1)可以看出, 合成虫草素的关键 是糖中间体 $\mathbf{3}$ 的合成，而化合物 $\mathbf{3}$ 可以通过 $D$-葡萄糖或 者 $D$-木糖合成得到. 首先考虑用廉价易得的 $D$-葡萄糖 为起始原料, 公斤级的双异亚丙基- $D$ - 葡萄糖 4 可以高 产率合成得到 ${ }^{[8]}$, 该化合物也可从商业以便宜的价格获 取; 双异亚丙基- $D$-匍萄糖 4 在 $\mathrm{NaH}$ 作用下和 $\mathrm{CS}_{2}$ 以及 $\mathrm{CH}_{3} \mathrm{I}$ 反应以 $95 \%$ 产率得到二硫代碳酸甲酯类糖 $\mathbf{5}^{[9]}$; 以

*E-mail: xiaoqiang@tsinghua.org.cn

Received March 6, 2013; revised April 24, 2013; published online May 6, 2013.

Project supported by the National Natural Science Foundation of China (NSFC, Nos. 20962009, 21062006), the Program for New Century Excellent Talents in University (NCET, No. 11-1000), the Training Project of Jiangxi Youth Scientists, and the Bureau of Science \& Technology of Nanchang City.

国家自然科学基金(NSFC, Nos. 20962009, 21062006)、教育部新世纪优秀人才支持计划(NCET, No. 11-1000)、江西省青年科学家人才计划和南昌市科 技局资助项目. 

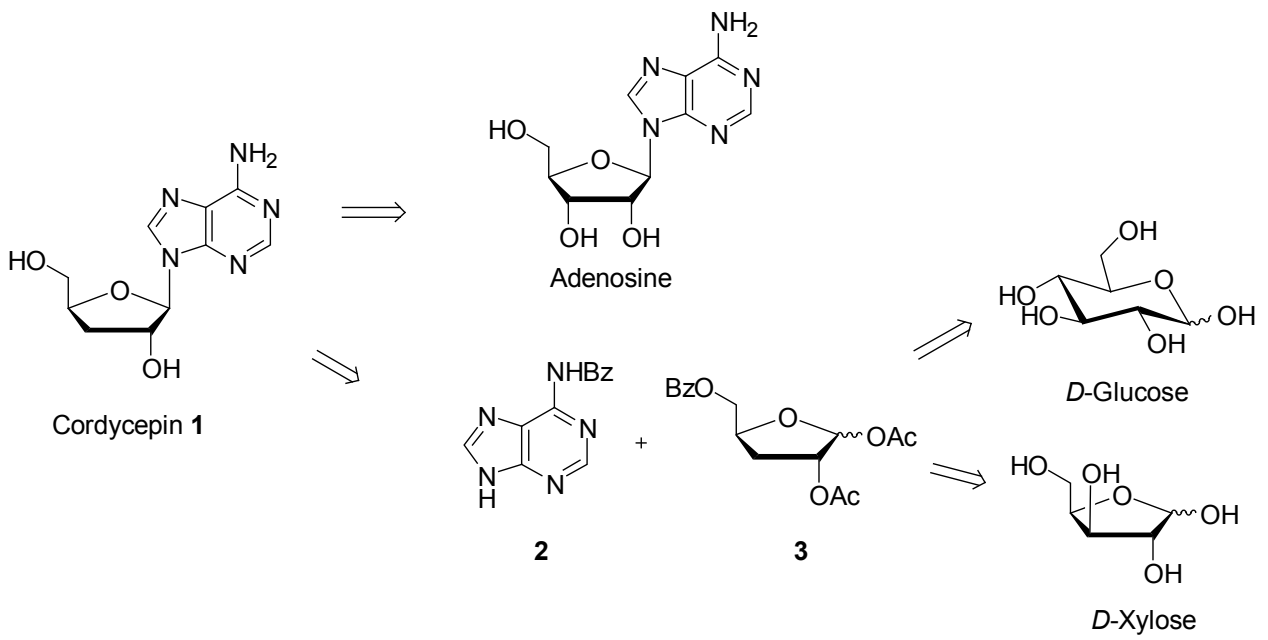

图 1 虫草素的结构及其反合成分析

Figure 1 Structure of Cordycepin and its retrosynthetic analysis

精制甲苯为溶剂, 在偶氮二异丁腈(AIBN)的引发下以 三正丁基氢化锡为还原剂经 Barton-McCombie 自由基还 原 ${ }^{[10]}$ 得到 3-脱氧双异亚丙基呋喃葡萄糖 $\mathbf{6}$, 经过多次反 应条件的优化, 产率可以稳定在 $80 \%$ 以上.

根据文献[11]报道，从 3-脱氧双异亚丙基- $D$-呋喃葡 萄糖 6 到 3-脱氧核糖 7 有两种可能的方法, 一种是先选 择性水解 5,6 的异亚丙基基团, 经过高碘酸氧化邻二醇, 然后经 $\mathrm{NaBH}_{4}$ 还原获得; 另一种是采用高碘酸原位水 解、氧化和还原一锅法制备 3-脱氧核糖 7. 为了提高反 应效率, 我们采用第二种方法. 在合成过程中我们发现, 反应中间体醛的稳定性较差, 而且是以水合物的形式出 现, 经过反应优化, 最终我们可以以接近 $80 \%$ 的产率得 到化合物 7, 但是反应放量到 $10 \mathrm{~g}$ 以上时, 反应产率迅 速下降至 $40 \%$ 左右. 经过多次尝试优化反应条件, 我们 仍然无法解决这个问题, 可能的原因是中间体醛基使得 核糖 4 位的氢有一定酸性, 导致异构化等副反应发生, 进而我们转向以 $D$-木糖为原料进行目标分子的合成.

在我们课题组前期的工作中 ${ }^{[12]}, 1,2-O$-异亚丙基$\alpha$ - $D$-木糖 8 可以以 $80 \%$ 产率从 $D$-木糖一锅法合成得到, 反应条件温和, 很容易放量到百克级. 接着以二氯甲烷 为溶剂, $\mathrm{Et}_{3} \mathrm{~N}$ 为碱, 低温下选择性合成 5- $O$-苯甲酰基1,2- $O$-异亚丙基- $\alpha$ - $D$-木糖 9, 产率可以稳定在 $80 \%$ 以 上 ${ }^{[13]}$. 单苯甲酰化产物 9 和硫代氯甲酸- $O$-苯酯反应很 顺利以 $86 \%$ 产率得到化合物 $\mathbf{1 0}$, 然后采用与合成化合 物 6 类似的 Barton-McCombie 自由基还原条件很顺利地 得到 3-脱氧核糖 11. 在硫酸催化下, 以醋酸和醋酐为溶 剂(10：1)脱去异亚丙基保护基团的同时乙酰化得到关 键中间体 3 , 产率 $84 \%$. 该方法容易放大, 产率稳定, 反 应后处理相对简单.

在 Vorbrüggen 糖基化条件下 ${ }^{[14]}$, 糖受体 3 和 $N^{6}$-苯
甲酰基腺嘌呤可以顺利以 $82 \%$ 产率得到核苷 12 , 由于 2 位乙酰基的邻基参与效应，反应仅得到 $\beta$ 糖苷化产物， HPLC 检测没有发现 $\alpha$ 糖苷化产物的生成. 最后在新制 备饱和氨水的甲醇溶液中, $110{ }^{\circ} \mathrm{C}$ 封管加热可以顺利脱 去所有酯基保护基团以 95\%产率得到虫草素，产物经过 水重结晶后 HPLC 分析纯度超过 $98.5 \%$.

\section{2 结论}

本文设计和完成了分别以 $D$-葡萄糖和 $D$-木糖为起 始原料, 以 Barton-McCombie 反应脱去葡糖糖和木糖 3'羟基合成得到 $3^{\prime}$-脱氧核糖为关键步骤，分别经过 8 步和 7 步反应完成了虫草素的全合成，总收率可以达到 $37 \% \sim 40 \%$, 对反应条件的进一步优化可以发展工业合 成虫草素的工艺, 这些工作正在进一步进行中.

\section{3 实验部分}

\section{1 仪器与试剂}

北京泰兴有限公司生产的 X-4 数字显示显微熔点仪 (温度计未校正); Bruker Advance DPX 400MHz 核磁共 振仪, 以 $\mathrm{TMS}$ 为内标, 忥代氯仿 $\left(\mathrm{CDCl}_{3}\right)$ 或者氝代二甲 亚砜(DMSO- $\left.d_{6}\right)$; 电喷雾质谱(ESI-MS)为 Bruker Amazon SL 离子阱质谱仪(正离子模式); 旋光测定使用美国 鲁道夫 Autopol IV-T 旋光仪; 紫外为 Agilent 8453 紫外 仪. 常规试剂从 Alfa 公司购买. 所有溶剂均为分析纯, 乙腈、吡啶和二氯甲烷使用前用氢化钲回流干燥, 使用 前蒸出; 甲苯经金属钠回流干燥, 使用前蒸出. 薄层色 谱(TLC)使用青岛化学工业公司的薄层板(GF-254), 在 紫外 $254 \mathrm{~nm}$ 波长或者用 $10 \%$ 硫酸显色进行检测. 硅胶 柱色谱使用青岛化学工业公司的硅胶(200～300 目). 

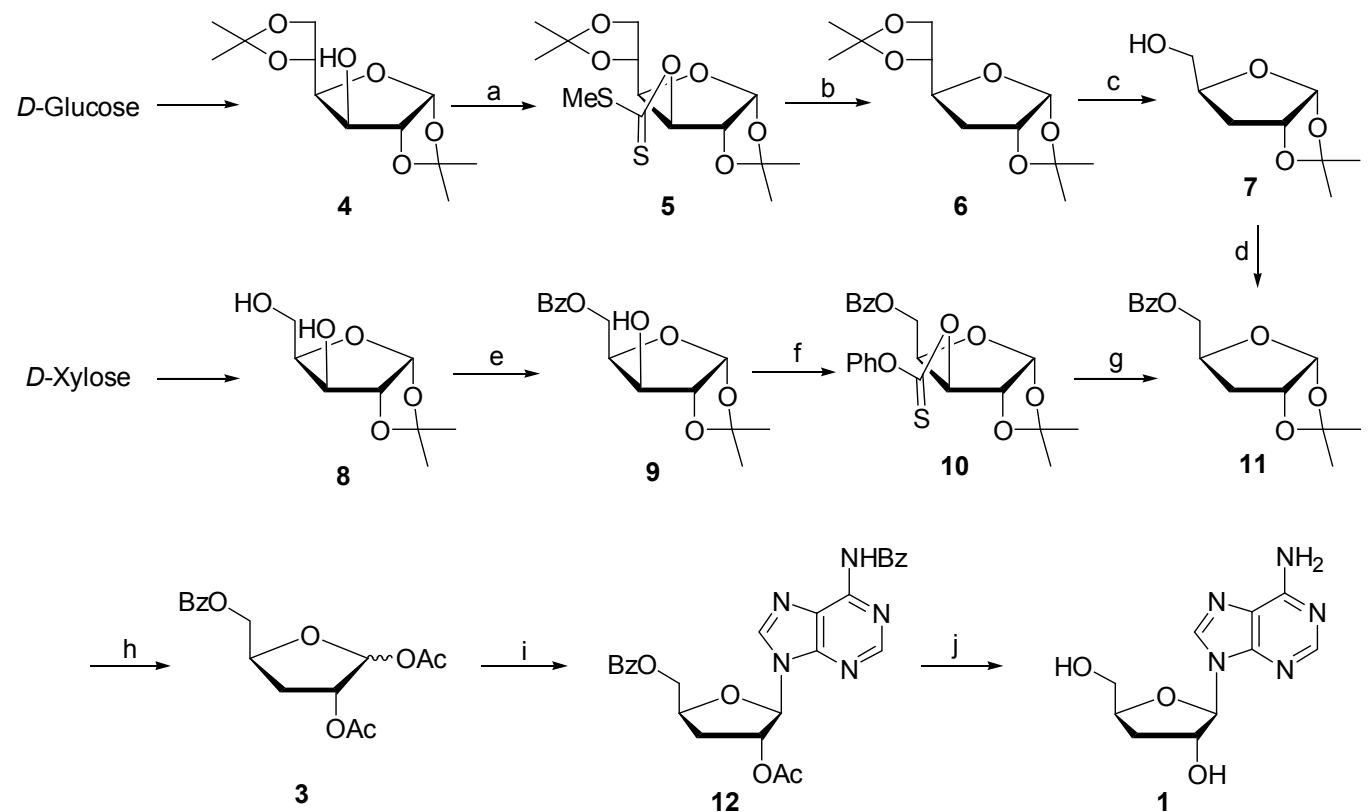

Reagents and conditions: (a) $\mathrm{NaH}$, imidazole, $\mathrm{CS}_{2}$, Mel, THF, r.t., $4 \mathrm{~h}, 95 \%$; (b) $\mathrm{Bu}_{3} \mathrm{SnH}$, AlBN, toluene, reflux, $110{ }^{\circ} \mathrm{C}, 2 \mathrm{~h}, 80 \%$; (c) i) $\mathrm{H}_{5} \mathrm{IO}{ }_{6}$, EtOAc; ii) $\mathrm{NaBH}_{4}$, EtOH, r.t., 1 h, $79 \%$; (d) $\mathrm{BzCl}_{2} \mathrm{Et}_{3} \mathrm{~N}$, DCM, $\mathrm{N}_{2}$, r.t., 2 h, $95 \%$; (e) $\mathrm{BzCl}, \mathrm{Et}_{3} \mathrm{~N}$, DCM, r.t., $\mathrm{N}_{2}, 1$ h, $88 \%$; (f) O-phenyl chlorothionocarbonate, DMAP, DCM, $\mathrm{N}_{2}$, r.t., 4 h, $86 \%$; (g) $\mathrm{Bu}_{3} \mathrm{SnH}$, AlBN, toluene, reflux, $110{ }^{\circ} \mathrm{C}, 2 \mathrm{~h}, 82 \%$; (h) $\mathrm{AcOH}, \mathrm{AcOAc} \mathrm{H}_{2} \mathrm{SO}{ }_{4}$, r.t., 6 h, $84 \%$; (i) BSA, TMSOTf, MeCN, reflux, $80{ }^{\circ} \mathrm{C}, 1 \mathrm{~h}, 82 \%$; (j) $7 \mathrm{~N} \mathrm{NH}_{3} / \mathrm{MeOH}, 110{ }^{\circ} \mathrm{C}, 8 \mathrm{~h}, 95 \%$

\section{Scheme 1}

\section{2 实验方法}

3.1.25,6-二- $O$ - 异亚丙基 $-3-O-($ 二硫代碳酸甲 酯) $-\alpha-D$-呋喃葡萄糖(5)的合成

将化合物 $4(50 \mathrm{~g}, 0.19 \mathrm{~mol})$ 和 $50 \mathrm{mg}$ 咪唑溶于 800 $\mathrm{mL}$ 无水 $\mathrm{THF}$ 中, 缓慢加入 $\mathrm{NaH}(50 \mathrm{~g}, 0.19 \mathrm{~mol})$, 氮气 保护下搅拌 $20 \mathrm{~min}$ 后,一次性加入 $\mathrm{CS}_{2}(43.88 \mathrm{~g}, 0.57$ $\mathrm{mol})$, 继续搅拌 $30 \mathrm{~min}$ 后, 缓慢滴加 $\mathrm{MeI}(49.08 \mathrm{~g}, 0.35$ $\mathrm{mol}), 3 \mathrm{~h}$ 后滴加完毕, 加完后继续反应 $15 \mathrm{~min}$ 后加入 10 $\mathrm{mL}$ 醋酸中和之前未反应完的 $\mathrm{NaH}$, 过滤, 滤液浓缩后 溶于 $500 \mathrm{~mL}$ 乙醚中, 分别用饱和 $\mathrm{NaHCO}_{3}(200 \mathrm{~mL} \times 3)$, 饱和食盐水 $(200 \mathrm{~mL} \times 2)$ 洗涤, 收集有机相用无水 $\mathrm{Na}_{2} \mathrm{SO}_{4}$ 干燥 $3 \mathrm{~h}$ 后蒸干, 残余物通过硅胶层析柱分离得 浅橙黄色粘稠状液体 $\mathbf{5}^{[9]}(64 \mathrm{~g}, 95 \%) ; R_{\mathrm{f}} 0.6$ (石油醚/乙 酸乙酯, $V: V=5: 1) ;{ }^{1} \mathrm{H}$ NMR $\left(400 \mathrm{MHz}, \mathrm{CDCl}_{3}\right) \delta$ : $5.91 \sim 5.89$ (m, 2H, H-1,3), 4.66 (d, J=4.6 Hz, 1H, H-2), $4.33 \sim 4.04$ (m, 4H, H-5,6a,4,6b), 2.57 (s, 3H, SMe), 1.52, $1.40,1.31,1.30(4 \mathrm{~s}, 12 \mathrm{H}, \mathrm{CMe}) ;{ }^{13} \mathrm{C}$ NMR $(100 \mathrm{MHz}$, $\left.\mathrm{CDCl}_{3}\right) \delta$ : $214.7(\mathrm{C}=\mathrm{S}), 112.4,109.3$ (2C, 2-异亚丙基碳), 105.0 (C-1), 84.2 (C-2), 82.8 (C-4), 79.8 (C-5), 72.4 (C-3), 66.9 (C-6), 26.8, 26.6, 26.2, 25.2 (4C, 4Me), 19.3 (SMe); ESI-MS $m / z: 373.0[\mathrm{M}+\mathrm{Na}]^{+}$.

3.2.2 3-脱氧-1,2:5,6-二- $O$-异亚丙基- $\alpha$ - $D$-呋喃葡萄 糖(6)的合成

将化合物 $5(20 \mathrm{~g}, 57.0 \mathrm{mmol})$ 和 AIBN (2.81 g, 17.1 $\mathrm{mmol}$ )溶解于 $250 \mathrm{~mL}$ 无水甲苯中, 常温摚拌 $10 \mathrm{~min}$, 通
氮气, 之后加入三正丁基氢化锡 $(21.6 \mathrm{~g}, 74.2 \mathrm{mmol})$ 并将 体系转移到 $110{ }^{\circ} \mathrm{C}$ 油浴中, 回流反应 $2 \mathrm{~h}$, 反应结束后, 将体系冷却到室温, 另外加入 $200 \mathrm{~mL}$ 乙酸乙酯, 分别 用 $10 \%$ 的 $\mathrm{KF}$ 水溶液 $(200 \mathrm{~mL} \times 3)$, 饱和食盐水 $(200$ $\mathrm{mL} \times 2$ ) 洗涤, 有机相用无水 $\mathrm{Na}_{2} \mathrm{SO}_{4}$ 干燥 $3 \mathrm{~h}$ 后蒸干, 残 余物通过硅胶柱层析分离得无色粘稠状液体 $\mathbf{6}^{[9]}(11.2 \mathrm{~g}$, $80 \%$ ). $R_{\mathrm{f}} 0.5$ (石油醚/乙酸乙酯, $V: V=4: 1$ ); ${ }^{1} \mathrm{H}$ NMR $\left(400 \mathrm{MHz}, \mathrm{CDCl}_{3}\right) \delta: 5.75$ (s, 1H, H-1), 4.69 (d, $J=4 \mathrm{~Hz}$, $1 \mathrm{H}, \mathrm{H}-2), 4.15 \sim 4.01(\mathrm{~m}, 3 \mathrm{H}, \mathrm{H}-5,6 \mathrm{a}, 4), 3.76 \sim 3.74(\mathrm{~m}$, $1 \mathrm{H}, \mathrm{H}-6 \mathrm{~b}), 2.12 \sim 2.09(\mathrm{~m}, 1 \mathrm{H}, \mathrm{H}-3 \mathrm{a}), 1.73 \sim 1.71(\mathrm{~m}, 1 \mathrm{H}$, $\mathrm{H}-3 \mathrm{~b}), 1.44,1.35,1.28,1.25$ (4s, 12H, 4CMe); ${ }^{13} \mathrm{C}$ NMR $\left(100 \mathrm{MHz}, \mathrm{CDCl}_{3}\right) \delta: 111.2,109.5$ (2C, 2 个异亚丙基季 碳), 105.5 (C-1), 80.3 (C-2), 78.5 (C-4), 76.7 (C-5), 67.1 (C-6), 35.1 (C-3), 26.7, 26.4, 26.1, 25.1 (4C, 4Me); ESI-MS $m / z: 267.0[\mathrm{M}+\mathrm{Na}]^{+}$.

\subsubsection{3-脱氧-1,2- $O$-异亚丙基- $\alpha$ - $D$-核糖(7)的合成}

将化合物 6 (8.5 g, $34.8 \mathrm{mmol}$ ) 溶解于 $400 \mathrm{~mL}$ 无水乙 酸乙酯中, 常温摚拌均匀, 加入高碘酸 $(9.5 \mathrm{~g}, 41.7$ $\mathrm{mmol})$, 继续摚拌, 体系颜色逐渐由无色变为橙黄色并 伴随有大量白色沉淀，原料反应完后立刻过滤，滤液蒸 干, 溶解于 $500 \mathrm{~mL}$ 乙醇中, 激烈搅拌下缓慢加入 $\mathrm{NaBH}_{4}(1.9 \mathrm{~g}, 52.1 \mathrm{mmol})$, 体系由浅黄色变为深棕色最 后变为乳白色半透明状, $1 \mathrm{~h}$ 后停止反应, 直接蒸干, 残 余物溶解于 $300 \mathrm{~mL}$ 乙酸乙酯中, 分别用饱和 $\mathrm{NaHCO}_{3}$ $(100 \mathrm{~mL} \times 3)$, 饱和食盐水 $(100 \mathrm{~mL} \times 2)$ 洗涤, 收集有机 
相用无水 $\mathrm{Na}_{2} \mathrm{SO}_{4}$ 干燥 $3 \mathrm{~h}$ 后蒸干, 残余物通过硅胶层析 柱分离得白色晶体 $\boldsymbol{7}^{[15]}(5.0 \mathrm{~g}, 79 \%) . R_{\mathrm{f}} 0.2$ (石油醚/乙酸 乙酯, $V: V=4: 1) ;$ m.p. $65 \sim 66{ }^{\circ} \mathrm{C} ;{ }^{1} \mathrm{H}$ NMR $(400$ $\left.\mathrm{MHz}, \mathrm{CDCl}_{3}\right) \delta: 5.82(\mathrm{~d}, J=3.5 \mathrm{~Hz}, 1 \mathrm{H}, \mathrm{H}-1), 4.76 \sim 4.74$ (m, 1H, H-2), $4.41 \sim 4.27(\mathrm{~m}, 1 \mathrm{H}, \mathrm{H}-4), 3.90 \sim 3.87(\mathrm{~m}$, 1H, H-5a), $3.57 \sim 3.54$ (m, 1H, H-5b), $2.01 \sim 1.98(\mathrm{~m}, 1 \mathrm{H}$, $\mathrm{H}-3 \mathrm{a}), 1.87 \sim 1.86(\mathrm{~m}, 1 \mathrm{H}, \mathrm{H}-3 \mathrm{~b}), 1.50,1.31(2 \mathrm{~s}, 6 \mathrm{H}$, $2 \mathrm{CMe}$ ); ${ }^{13} \mathrm{C} \mathrm{NMR}\left(100 \mathrm{MHz}, \mathrm{CDCl}_{3}\right.$ ) $\delta: 111.3$ (异亚丙基 碳), 105.5 (C-1), 80.8 (C-2), 78.5 (C-4), 62.9 (C-5), 33.8 (C-3), 26.8, 26.2 (2C, 2Me); ESI-MS m/z: $197.1[\mathrm{M}+$ $\mathrm{Na}]^{+}$.

3.2.45-O-苯甲酰基-3-脱氧-1,2- $O$-异亚丙基- $\alpha$ - $D$-核 糖(11)的合成

将化合物 7 (5.0 g, $28.7 \mathrm{mmol})$ 溶解于无水二氯甲烷 $(30 \mathrm{~mL})$ 中, $0{ }^{\circ} \mathrm{C}$ 搅拌, 充氮气 $10 \mathrm{~min}$, 加入无水三乙胺 (约 $3 \mathrm{~mL}$ ), 缓慢滴加苯甲酰氯 $(4.94 \mathrm{~g}, 4.8 \mathrm{~mL}, 31.57$ $\mathrm{mmol}$ ), 反应 $2 \mathrm{~h}$ 后, TLC 检测结果表明原料反应完全, 加少量甲醇淬灭反应, 减压浓缩, 残余物通过硅胶柱层 析分离得无色油状物 $\mathbf{1 1}^{[16]}(7.6 \mathrm{~g}, 95 \%)$ ). $R_{\mathrm{f}} 0.45$ (石油醚/ 乙酸乙酯, $V: V=4: 1) ;{ }^{1} \mathrm{H} \mathrm{NMR}\left(400 \mathrm{MHz}, \mathrm{CDCl}_{3}\right) \delta$ : $8.04 \sim 7.42$ (m, 5H, Ar), $5.86(\mathrm{~d}, J=3.6 \mathrm{~Hz}, 1 \mathrm{H}, \mathrm{H}-1)$, $4.77 \sim 4.75(\mathrm{~m}, 1 \mathrm{H}, \mathrm{H}-2), 4.54 \sim 4.53(\mathrm{~m}, 1 \mathrm{H}, \mathrm{H}-5 \mathrm{a})$, $4.51 \sim 4.50(\mathrm{~m}, 1 \mathrm{H}, \mathrm{H}-4), 4.37 \sim 4.35(\mathrm{~m}, 1 \mathrm{H}, \mathrm{H}-5 \mathrm{~b})$, $2.19 \sim 2.14(\mathrm{~m}, 1 \mathrm{H}, \mathrm{H}-3 \mathrm{a}), 1.78 \sim 1.74(\mathrm{~m}, 1 \mathrm{H}, \mathrm{H}-3 \mathrm{~b})$, 1.52, $1.32\left(2 \mathrm{~s}, 6 \mathrm{H}, \mathrm{CMe}_{2}\right) ;{ }^{13} \mathrm{C} \mathrm{NMR}\left(100 \mathrm{MHz}, \mathrm{CDCl}_{3}\right) \delta$ : $166.2(\mathrm{C}=\mathrm{O}), 133.0,129.7,128.3 \times 2(4 \mathrm{C}, \mathrm{Ar}), 111.2$ (异 亚丙基碳), 105.7 (C-1), 80.2 (C-2), 75.8 (C-4), 65.2 (C-5), 35.4 (C-3), 26.7, 26.1 (2C, Me); ESI-MS m/z: $301.1[\mathrm{M}+$ $\mathrm{Na}]^{+}$.

3.2.5 5- $O$-苯甲酰基-1,2- $O$-异亚丙基- $\alpha-D$-木糖(9)的 合成

将化合物 $8(11 \mathrm{~g}, 57.84 \mathrm{mmol})$ 溶解于无水二氯甲烷 $(70 \mathrm{~mL})$ 中, $0{ }^{\circ} \mathrm{C}$ 搅拌, 充氮气置换体系里的空气 $(10$ $\mathrm{min}$ ), 加入无水三乙胺(约 $3 \mathrm{~mL}$ ), 缓慢滴加新蒸的苯甲 酰氯( $8.54 \mathrm{~g}, 7.05 \mathrm{~mL}, 60.73 \mathrm{mmol})$, 反应 $2 \mathrm{~h}$ 后, TLC 检 测显示原料消失, 加甲醇 $(1 \mathrm{~mL})$ 淬灭反应, 减压浓缩除 去溶剂, 残余物通过硅胶柱层析分离纯化, 得无色油状 物 $\mathbf{9}^{[17]}(15.0 \mathrm{~g}, 88 \%) ; R_{\mathrm{f}} 0.2$ (石油醚/乙酸乙酯, $V: V=$ $5: 1) ;{ }^{1} \mathrm{H}$ NMR $\left(400 \mathrm{MHz}, \mathrm{CDCl}_{3}\right) \delta: 8.05 \sim 7.44(\mathrm{~m}, 5 \mathrm{H}$, Ar), $5.96(\mathrm{~d}, J=3.6 \mathrm{~Hz}, 1 \mathrm{H}, \mathrm{H}-1), 4.76 \sim 4.35(\mathrm{~m}, 4 \mathrm{H}$, H-2,4,5a,b), 4.21 (d, $J=1.6 \mathrm{~Hz}, 1 \mathrm{H}, \mathrm{H}-3), 1.52,1.32(2 \mathrm{~s}$, $6 \mathrm{H}, 2 \mathrm{CMe}) ;{ }^{13} \mathrm{C} \mathrm{NMR}\left(100 \mathrm{MHz}, \mathrm{CDCl}_{3}\right) \delta: 167.3(\mathrm{C}=$ O), 133.5, 129.8, 128.6, 128.5 (4C, Ar), 111.8 (异亚丙基 碳), 104.7 (C-1), 85.0 (C-2), 78.5 (C-4), 74.5 (C-3), 61.6 (C-5), 26.7, 26.1 (2C, CMe); ESI-MS $m / z: 317.1[\mathrm{M}+$
$\mathrm{Na}]^{+}$.

3.2.6 5- $O$ - 苯甲酰基-3- $O$ - 硫代苯甲酰基-1,2- $O$-异亚 丙基- $\alpha$ - $D$-木糖(10)的合成

将化合物 9 (10.0 g, $33.9 \mathrm{mmol})$ 溶于无水二氯甲烷 (200 mL)中, 加入 DMAP $(10.4 \mathrm{~g}, 84.9 \mathrm{mmol})$ 和硫代氯 甲酸- $O$-苯酯 $(7.1 \mathrm{~g}, 40.8 \mathrm{mmol})$, 在氮气保护下, 常温摚 拌 $2 \mathrm{~h}$, 然后分别用 $0.5 \mathrm{~mol} / \mathrm{L}$ 的盐酸 $(80 \mathrm{~mL} \times 2), 0.5$ $\mathrm{mol} / \mathrm{L}$ 氢氧化钠 $(80 \mathrm{~mL} \times 2)$, 水 $(80 \mathrm{~mL} \times 2)$, 饱和食盐水 $(80 \mathrm{~mL} \times 2)$ 洗涤, 有机相用无水硫酸钠干燥 $3 \mathrm{~h}$, 减压浓 缩, 残余物通过硅胶柱层析分离, 得浅黄色油状化合物 $10^{[18]}\left(12.6 \mathrm{~g}, 86 \%\right.$ ). $R_{\mathrm{f}} 0.6$ (石油醚/乙酸乙酯, $V: V=5$ : 1); ${ }^{1} \mathrm{H}$ NMR $\left(400 \mathrm{MHz}, \mathrm{CDCl}_{3}\right) \delta: 8.19 \sim 7.99(\mathrm{~m}, 10 \mathrm{H}$, Ar), $6.10(\mathrm{~d}, J=3.6 \mathrm{~Hz}, 1 \mathrm{H}, \mathrm{H}-1), 5.86(\mathrm{~d}, J=2.8 \mathrm{~Hz}, 1 \mathrm{H}$, $\mathrm{H}-3), 4.85 \sim 4.79(\mathrm{~m}, 1 \mathrm{H}, \mathrm{H}-2), 4.79 \sim 4.77(\mathrm{~m}, 1 \mathrm{H}, \mathrm{H}-4)$, $4.74 \sim 4.55(\mathrm{~m}, 2 \mathrm{H}, \mathrm{H}-5 \mathrm{a}, \mathrm{b}), 1.52,1.32\left(2 \mathrm{~s}, 6 \mathrm{H}, \mathrm{CMe}_{2}\right)$; ${ }^{13} \mathrm{C}$ NMR $\left(100 \mathrm{MHz}, \mathrm{CDCl}_{3}\right) \delta: 192.9(\mathrm{C}=\mathrm{S}), 165.1(\mathrm{C}=$ O), 132.2 120.6 (8C, 2Ar), 111.5 (异亚丙基碳), 104.0 (C-1), 84.1 (C-2), 82.1 (C-4), 75.8 (C-3), 60.5 (C-5), 25.7, 25.2 (2Me); ESI-MS $m / z: 491.1[\mathrm{M}+\mathrm{Na}]^{+}$.

3.2.75-O-苯甲酰基-3-脱氧-1,2- $O$-异亚丙基- $\alpha$ - $D$-核 糖(11)的合成

将化合物 $\mathbf{1 0}$ (10.0 g, $23.23 \mathrm{mmol}$ )溶于无水甲苯(300 $\mathrm{mL})$ 中, 加入新重结晶的 $\operatorname{AIBN}(0.77 \mathrm{~g}, 4.7 \mathrm{mmol})$, 常温 下充氮气 $20 \mathrm{~min}$, 然后加入三正丁基氢化锡 $(9.2 \mathrm{~g}, 31.4$ $\mathrm{mmol}$ ), 之后移入事先预热好的 $110{ }^{\circ} \mathrm{C}$ 油浴中回流 $2 \mathrm{~h}$, 冷却到室温, 经过 $\mathrm{KF}$ 和硅藻土的混合物过滤, 滤液减 压浓缩, 残余物硅胶柱层析分离纯化的油状纯净物 $11^{[16]}(5.3 \mathrm{~g}, 82 \%) . R_{\mathrm{f}} 0.45$ (石油醚/乙酸乙酯, $V: V=4$ : 1); ${ }^{1} \mathrm{H}$ NMR (400 MHz, $\left.\mathrm{CDCl}_{3}\right) \delta: 8.04 \sim 7.42$ (m, 5H, Ar), $5.86(\mathrm{~d}, J=3.6 \mathrm{~Hz}, 1 \mathrm{H}, \mathrm{H}-1), 4.77 \sim 4.75(\mathrm{~m}, 1 \mathrm{H}, \mathrm{H}-2)$, $4.56 \sim 4.53(\mathrm{~m}, 1 \mathrm{H}, \mathrm{H}-5 \mathrm{a}), 4.51 \sim 4.50(\mathrm{~m}, 1 \mathrm{H}, \mathrm{H}-4)$, $4.37 \sim 4.33(\mathrm{~m}, 1 \mathrm{H}, \mathrm{H}-5 \mathrm{~b}), 2.19 \sim 2.14(\mathrm{~m}, 1 \mathrm{H}, \mathrm{H}-3 \mathrm{a})$, $1.78 \sim 1.74(\mathrm{~m}, 1 \mathrm{H}, \mathrm{H}-3 \mathrm{~b}), 1.52,1.32\left(2 \mathrm{~s}, 6 \mathrm{H}, \mathrm{CMe}_{2}\right) ;{ }^{13} \mathrm{C}$ NMR $\left(100 \mathrm{MHz}, \mathrm{CDCl}_{3}\right) \delta: 166.2(\mathrm{C}=\mathrm{O}), 133.0,129.7$, $128.3 \times 2$ (4C, Ar), 111.2 (异亚丙基碳), 105.7 (C-1), 80.2 (C-2), 75.8 (C-4), 65.2 (C-5), 35.4 (C-3), 26.7, 26.1 (2C, $\mathrm{Me}) ; \mathrm{ESI}-\mathrm{MS} m / z: 301.1[\mathrm{M}+\mathrm{Na}]^{+}$.

3.2.8 1,2-二- $O$-乙酰基-5- $O$ - 苯甲酰基-3-脱氧- $D$-核 糖(3)的合成

将化合物 11 (4.0 g, $14.4 \mathrm{mmol})$ 冰水浴冷却下加入 到含有硫酸 $(2.0 \mathrm{~mL})$ 、醋酸 $(80.0 \mathrm{~mL})$ 、醋酎 $(8.0 \mathrm{~mL})$ 的混 合液中, 常温摚拌约 $6 \mathrm{~h}, \mathrm{TLC}$ 检测反应结束, 加 100.0 $\mathrm{mL}$ 冰水稀释, 用二氯甲烷 $(200 \mathrm{~mL})$ 萃取, 分别用饱和 碳酸氢钠 $(80 \mathrm{~mL} \times 3)$, 饱和食盐水 $(80 \mathrm{~mL} \times 2)$ 洗涤, 有 机相用无水硫酸钠干燥 $3 \mathrm{~h}$, 减压浓缩, 残余物硅胶柱 
层析分离纯化得无色油状物 $\mathbf{3}^{[16]}(3.9 \mathrm{~g}, 84 \%, \beta: \alpha=$ $8: 1$ ); $R_{\mathrm{f}} 0.3$ (石油醚/乙酸乙酯, $V: V=5: 1$ ); $\beta$ 异构体 核磁数据为 ${ }^{1} \mathrm{H}$ NMR $\left(400 \mathrm{MHz}, \mathrm{CDCl}_{3}\right) \delta: 8.04,7.57 \sim$ $7.40(\mathrm{~m}, 5 \mathrm{H}, \mathrm{Ar}), 6.18(\mathrm{~s}, 1 \mathrm{H}, \mathrm{H}-1 \beta), 5.22 \sim 5.21(\mathrm{~m}, 1 \mathrm{H}$, $\mathrm{H}-2 \beta), 4.71 \sim 4.68(\mathrm{~m}, 1 \mathrm{H}, \mathrm{H}-4), 4.53 \sim 4.31(\mathrm{~m}, 2 \mathrm{H}$, $\mathrm{H}-5 \mathrm{a}, \mathrm{b}), 2.08$ (s, 3H, CMe), 1.94 (s, 3H, CMe); ${ }^{13} \mathrm{C} \mathrm{NMR}$ $\left(100 \mathrm{MHz}, \mathrm{CDCl}_{3}\right) \delta: 169.4,168.8,165.7(3 \mathrm{C}, 3 \mathrm{C}=\mathrm{O})$, 132.7, 129.2, 128.0, 127.9 (4C, Ar), 98.9 (C-1), 78.2 (C-2), 76.3 (C-4), 65.5 (C-5), 31.0 (C-3), 20.6, 20.4 (2C, Me); ESI-MS $m / z: 345.3[\mathrm{M}+\mathrm{Na}]^{+}$.

3.2.93-脱氧- $N^{6}$ - 苯甲酰基-9-(2'- $O$-乙酰基-5'- $O$ - 苯甲 酰基- $3^{\prime}$-脱氧 $-\beta-D$-核糖)腺苷(12)的合成

将化合物 $2(820 \mathrm{mg}, 3.4 \mathrm{mmol})$ 加入新蒸的无水 $1,2-$ 二氯乙烷 $(40 \mathrm{~mL})$ 中, 通氮气 $10 \mathrm{~min}$, 加入 BSA $(2.5 \mathrm{~g}$, $12.4 \mathrm{mmol}$ )摚拌直到碱基全部溶解 (10 min), 分别加入 化合物 3 (1.0 g, $3.1 \mathrm{mmol}$ ) 和 TMSOTf (4.1 g, $18.6 \mathrm{mmol})$, 常温摚拌 $15 \mathrm{~min}$, 将体系移入 $80{ }^{\circ} \mathrm{C}$ 油浴中, 反应 $2 \mathrm{~h}$, 减 压浓缩, 残余物硅胶柱层析分离纯化得产物 $\mathbf{1 2}^{[5]}(1.4 \mathrm{~g}$, $82 \%$ ). $R_{\mathrm{f}} 0.5$ (二氯甲烷/甲醇, $V: V=7 ： 1$ ); m.p. 78 81 ${ }^{\circ} \mathrm{C} ;[\alpha]_{\mathrm{D}}^{25}-10\left(c 0.1, \mathrm{CH}_{3} \mathrm{CN}\right) ;{ }^{1} \mathrm{H}$ NMR (400 MHz, $\mathrm{CDCl}_{3}$ ) $\delta: 9.55$ (s, 1H, NH), 8.69 (s, 1H, H-2), 8.12 (s, 1H, H-8), $8.03 \sim 7.82,7.54 \sim 7.34$ (m, 10H, 2Ar), 6.09 (s, 1H, H-1'), $5.82 \sim 5.81\left(\mathrm{~m}, 1 \mathrm{H}, \mathrm{H}-2^{\prime}\right), 4.70 \sim 4.50\left(\mathrm{~m}, 3 \mathrm{H}, \mathrm{H}-4^{\prime}, 5^{\prime}\right)$, $2.90 \sim 2.86\left(\mathrm{~m}, 1 \mathrm{H}, \mathrm{H}-3 \mathrm{a}^{\prime}\right), 2.32 \sim 2.29\left(\mathrm{~m}, 1 \mathrm{H}, \mathrm{H}-3 \mathrm{~b}^{\prime}\right)$, 2.14 (s, 3H, CMe); ${ }^{13} \mathrm{C}$ NMR (100 MHz, $\left.\mathrm{CDCl}_{3}\right) \delta 170.1$, 166.2, 164.6 (3C, C=O), 152.5 (C-6), $151.1(\mathrm{C}-2), 149.6$ (C-4), 141.9 (C-8), 133.6 127.9 (8C, 2Ar), 123.4 (C-5), 90.4 (C-1'), 78.9 (C-2'), 77.5 (C-4'), 64.8 (C-5'), 33.1 (C-3'), 20.8 (CMe); ESI-MS m/z: $524.1[\mathrm{M}+\mathrm{Na}]^{+}$.

\section{2 .10 虫草素(1)的合成}

将化合物 $12(800 \mathrm{mg}, 1.60 \mathrm{mmol})$ 溶于新制备的氨 气的甲醇饱和溶液中 $(15 \mathrm{~mL}), 110{ }^{\circ} \mathrm{C}$ 封管反应 $8 \mathrm{~h}$, 冷 却至室温, 减压浓缩, 残余物硅胶柱层析分离纯化得产 物 $\mathbf{1}^{[5]}$ (380 mg, 95\%). $R_{\mathrm{f}} 0.3$ (二氯甲烷/甲醇, $6: 1$ ); m.p. $229 \sim 232{ }^{\circ} \mathrm{C} ;[\alpha]_{\mathrm{D}}^{20}-40$ (c $\left.0.05, \mathrm{CH}_{3} \mathrm{OH}\right)$; UV-Vis $\left(\mathrm{CH}_{3} \mathrm{OH}\right) \lambda_{\max }: 213,260 \mathrm{~nm} ;{ }^{1} \mathrm{H}$ NMR (400 MHz, DMSO) $\delta: 8.35$ (s, $1 \mathrm{H}, \mathrm{H}-2), 8.12$ (s, $1 \mathrm{H}, \mathrm{H}-8), 7.31$ (s, $2 \mathrm{H}, \mathrm{NH}_{2}$ ), $5.85\left(\mathrm{~d}, J=5.8 \mathrm{~Hz}, 1 \mathrm{H}, \mathrm{OH}-1^{\prime}\right), 5.67(\mathrm{~d}, J=5.7 \mathrm{~Hz}, 1 \mathrm{H}$, OH-2'), 5.19 5.18 (m, 1H, OH-5'), 4.55 (s, 1H, H-2'), 4.33 (s, 1H, H-4'), $3.69 \sim 3.66$ (m, 1H, H-5a'), $3.51 \sim 3.46$ (m, 1H, H-5b'), $2.27 \sim 2.20$ (m, 2H, H-3a'), $1.93 \sim 1.89$ (m, $\left.2 \mathrm{H}, \mathrm{H}-3 \mathrm{~b}^{\prime}\right) ;{ }^{13} \mathrm{C}$ NMR (100 MHz, DMSO- $\left.d_{6}\right) \delta: 156.5$ (C-6), 152.8 (C-2), 149.3 (C-4), 139.5 (C-8), 119.5 (C-5), 91.2 (C-1'), 81.1 (C-2'), 75.0 (C-4'), 63.1 (C-5'), 34.5
(C-3'); ESI-MS m/z: $274.1[\mathrm{M}+\mathrm{Na}]^{+}$.

\section{References}

[1] (a) Bentley, H. R.; Cunningham, K. G.; Spring, F. S. J. Chem. Soc. 1951, 2301.

(b) Cunningham, K. G.; Manson, W.; Spring, F. S.; Hutchinson, S. A. Nature 1950, 166, 949.

[2] (a) Paterson, R. R. M. Phytochemistry 2008, 69, 1469. (b) Ng, T. B.; Wang, H. X. J. Pharm. Pharmacol. 2005, 57, 1509.

[3] Jeong, M.-H.; Seo, M. J.; Park, J. U.; Kang, B. W.; Kim, K.-S.; Lee, J. Y.; Kim, G.-Y.; Kim, J.-I.; Choi, Y. H.; Kim, K. H.; Jeong, Y. K. J. Microbiol. Biotechnol. 2012, 22, 1161.

[4] Ramesh, T.; Yoo, S. K.; Kim, S. W.; Hwang, S. Y.; Sohn, S. H.; Kim, I. W.; Kim, S. K. Exp. Gerontol. 2012, 47, 979.

[5] (a) Neumann, W. P.; Peterseim, M. Synlett 1992, 801 (b) Meier, C.; Huynh Dinh, T. Synlett 1991, 227.

(c) Kumar, A.; Khan, S. I.; Manglani, A.; Khan, Z. K.; Katti, S. B. Nucleosides Nucleotides 1994, 13, 1049.

[6] (a) Aman, S.; Anderson, D. J.; Connolly, T. J.; Crittall, A. J.; Ji, G. Org. Proc. Res. Develop. 2000, 4, 601.

(b) Robins, M. J.; Wilson, J. S.; Madej, D.; Low, N. H.; Hansske, F.; Wnuk, S. F. J. Org. Chem. 1995, 60, 7902.

[7] McDonald, F. E.; Gleason, M. M. J. Am. Chem. Soc. 1996, 118, 6648.

[8] Schmidt, O. T. Methods in Carbohydrate Chemistry, Vol. 2, Academic Press, New York, 1963, pp. 318 325.

[9] Iacono, S.; Rasmussen, J. R. Org. Synth. 1986, 64, 57.

[10] (a) Zard, S. Z. Aust. J. Chem. 2006, 59, 663.

(b) Chatgilialoglu, C.; Ferreri, C. Res. Chem. Inter. 1993, 19, 755.

(c) Barton, D. H. R.; McCombie, S. W. J. Chem. Soc., Perkin Trans. 1 1975, 1574.

(d) Han, S. H.; Qu, G. R.; Li, Y. Chin. J. Org. Chem. 2005, 25, 526 (in Chinese).

(韩素辉, 渠桂荣, 李永, 有机化学, 2005, 25, 526.)

[11] (a) Xie, M. Q.; Berges, D. A.; Robins, M. J. J. Org. Chem. 1996, 61,5178 .

(b) Wnuk, S. F.; Robert, J.; Sobczak, A. J.; Meyers, B. P.; Malladi, V. L. A.; Zhu, J.; Gopishetty, B.; Pei, D. Biol. Med. Chem. 2009, $17,6699$.

[12] Sun, J.; Dou, Y.; Ding, H.; Yang, R.; Sun, Q.; Xiao, Q. Mar. Drugs 2012, 10, 881.

[13] (a) Mathe, C.; Imbach, J. L.; Gosselin, G. Carbohydr. Res. 2000, $323,226$.

(b) Gosselin, G.; Puech, F.; Genudellac, C.; Imbach, J. L. Carbohydr. Res. 1993, 249, 1.

[14] (a) Niedballa, U.; Vorbruggen, H. J. Org. Chem. 1976, 41, 2084. (b) Vorbruggen, H.; Krolikiewicz K.; Bennua, B. Chem. Ber. 1981, 114, 1234 .

(c) Vorbrüggen, H.; Ruh-Pohlenz, C. Handbook of Nucleoside Synthesis, John Wiley \& Sons, New York, 2001.

[15] Shiozaki, M.; Deguchi, N.; Mochizuki, T.; Wakabayashi, T.; Ishikawa, T.; Haruyama, H.; Kawai, Y.; Nishijima, M. Tetrahedron 1998, 54,11861

[16] Kanda, S. R.; Robert, C. T.; Josie, B.; Devron, R. A. J. Med. Chem. 2000, 43, 1019.

[17] Christopher, J. W. M.; Marie, E. M.; Antony, G.; Barry, V. L. P. Bioorg. Med. Chem. 2004, 12, 475.

[18] Carmelo, J. R.; Joseph, P. D.; Ronald, B. Tetrahedron Lett. 1992, $33,4129$. 